\title{
A sufficient condition for counterexamples to the Nelson-Seiberg theorem
}

\author{
Zheng Sun, Zipeng Tan and Lu Yang \\ College of Physics, Sichuan University, \\ 29 Wangjiang Road, Chengdu 610064, P. R. China \\ E-mail: sun_ctp@scu.edu.cn, tzpcyc@126.com, 1546265328@qq.com
}

ABSTRaCT: Several counterexample models to the Nelson-Seiberg theorem have been discovered in previous literature, with generic superpotentials respecting the R-symmetry and non-generic R-charge assignments for chiral fields. This work present a sufficient condition for such counterexample models: the number of R-charge 2 fields, which is greater than the number of R-charge 0 fields, must be less than or equal to the number of R-charge 0 fields plus the number of independent field pairs with opposite R-charges and satisfying some extra requirements. We give a correct count of such field pairs when there are multiple field pairs with degenerated R-charges. These models give supersymmetric vacua with spontaneous R-symmetry breaking, thus are counterexamples to both the Nelson-Seiberg theorem and its extensions.

KEYwords: Global Symmetries, Supersymmetry Breaking

ArXiv EPrint: 2106.08879 


\section{Contents}

1 Introduction 1

2 The Nelson-Seiberg theorem and counterexamples 2

3 A sufficient condition for counterexamples 3

4 Examples of counterexamples $\quad 6$

5 Outlook $\quad 8$

\section{Introduction}

The Nelson-Seiberg theorem [1] and its extensions [2, 3] provide a method using R-symmetries to build F-term supersymmetry (SUSY) breaking vacua [4] in the framework of WessZumino models [5, 6] or O'Raifeartaigh models [7]. The theorem also applies to metastable SUSY breaking models [8] with approximate R-symmetries [9, 10]. SUSY preserving vacua can also be build with properly arranged R-charges of fields [11, 12]. These vacua appear in the model building of both SUSY phenomenology beyond the Standard Model [13-17] and compactification of string theory [18-22].

The proof of both the Nelson-Seiberg theorem and its extensions require generic superpotentials. The term genericness usually refers to generic values of the superpotential parameters. Non-generic models of this type require fine-tuning of parameters, and are not interesting for phenomenology studies. Another type of non-genericness comes from the specific form of the superpotential restricted by the R-symmetry with some special R-charge arrangement. Such non-generic models do not suffer from parameter tuning, and can be referred to as counterexamples to the Nelson-Seiberg theorem with non-generic R-charges. The first counterexample model of this type has been discovered recently [23] and thereafter generalized [24]. A common feature of these models is that they have at least one field pairs with opposite R-charges. The product of such field pairs act effectively like R-charge 0 fields for the field counts in the revised Nelson-Seiberg theorem [2], so SUSY vacua exist in these models even with more R-charge 2 fields than R-charge 0 fields. Following this idea, a sufficient condition for counterexamples is presented in [24], and new counterexamples are accordingly constructed using multiple field pairs with opposite R-charges.

In this work, we are to present a more general sufficient condition for counterexamples. In addition to the one in [24], we consider the case with multiple R-charge 2 and R-charge 0 fields. We also give a correct count of independent field pairs with opposite R-charges when there are multiple field pairs with degenerated R-charges. The sufficient condition is stated in terms of these field counts. The statement is then supported with examples which give 
SUSY vacua with spontaneous R-symmetry breaking, as predicted by field counts satisfying the sufficient condition.

The rest part of this paper is arranged as following. Section 2 reviews the NelsonSeiberg theorem, its extensions and previously found counterexamples. Section 3 presents the sufficient condition with a proof, and discusses properties of vacua from counterexamples. Section 4 gives examples supporting our statement. Section 5 makes the conclusion and final remarks.

\section{The Nelson-Seiberg theorem and counterexamples}

In a Wess-Zumino model, SUSY vacua are solutions to the F-term equations

$$
\partial_{i} W=\frac{\partial W}{\partial \phi_{i}}=0
$$

where $W=W\left(\phi_{i}\right)$, named the superpotential, is a holomorphic function of chiral superfields $\phi_{i}$ for $i=1, \ldots, n$. Such a solution also corresponds to a minimum of the scalar potential

$$
V=K^{\bar{i} j}\left(\partial_{i} W\right)^{*} \partial_{j} W
$$

with the vacuum expectation value (VEV) $\langle V\rangle=0$. Einstein summation is used in the expression of $V$, and the Kähler metric $K^{\overline{i j}}$ is calculated from a Kähler potential $K\left(\phi_{i}^{*}, \phi_{j}\right)$ which is a real and positive-definite function of $\phi_{i}$ 's and their conjugates $\phi_{i}^{*}$ 's. The vacuum is determined just by the scalar components $z_{i}$ 's of $\phi_{i}$ 's, so $W$ and $K$ are also viewed as functions of $z_{i}$ 's. If (2.1) has no solution, SUSY must be broken at any vacuum of the model, although we need to assume the existence of a local minimum of $V$, or build a minimum by introducing various corrections to a runaway direction [25-28]. Taking the non-existence of a solution to (2.1) as the criteria for SUSY breaking, the Nelson-Seiberg theorem and its extensions [1-3] give the conditions for SUSY breaking, expressed in terms of R-symmetries under which $W$ has R-charge 2. These theorems are as follows:

Theorem 1 (The Nelson-Seiberg theorem) In a Wess-Zumino model with a generic superpotential, assuming the existence of a vacuum at the global minimum of the scalar potential, an R-symmetry is a necessary condition, and a spontaneously broken R-symmetry is a sufficient condition for SUSY breaking at the vacuum.

Theorem 2 (The Nelson-Seiberg theorem revised and generalized) In a Wess-Zumino model with a generic superpotential, assuming the existence of a vacuum at the global minimum of the scalar potential, SUSY is spontaneously broken at the vacuum if and only if the superpotential has an R-symmetry, and one of the following conditions is satisfied:

- The superpotential is smooth at the origin of the field space, and the number of $R$ charge 2 fields is greater than the number of $R$-charge 0 fields for any possible consistent $R$-charge assignment.

- The superpotential is singular at the origin of the field space. 
For a generic R-symmetric polynomial $W$, the criteria for SUSY breaking is just the comparison between R-charge 2 and R-charge 0 field counts, which can be done much more easily and quickly than solving the F-term equations (2.1). Thus it is possible to do a fast scan of a large number of models based on the field counting criteria. The accuracy of such a scan is affected by the existence of counterexamples with generic parameters and non-generic R-charges. The simplest counterexample model [23] of this type has four fields $\left\{z_{1}, z_{2}, z_{3}, z_{4}\right\}$ with R-charges

$$
\left(R\left(z_{1}\right), R\left(z_{2}\right), R\left(z_{3}\right), R\left(z_{4}\right)\right)=(2,-2,6,-6),
$$

and the superpotential

$$
W=a z_{1}+b z_{1}^{2} z_{2}+c z_{2}^{2} z_{3}+d z_{1} z_{3} z_{4}
$$

which includes all renormalizable terms of R-charge 2 . The R-charge assignment is also uniquely fixed by requiring all terms of $W$ to have $\mathrm{R}$-charge 2 . The model has one $\mathrm{R}$-charge 2 field $z_{1}$ and no R-charge 0 field, so the field counting criteria predicts SUSY breaking. But the F-term equations have a SUSY solution

$$
z_{1}=z_{2}=0, \quad z_{3} z_{4}=-\frac{a}{d}
$$

which also breaks the R-symmetry for generic non-zero values of parameters $a$ and $d$. Thus the model is a counterexample to both the original and the revised Nelson-Seiberg theorems. The counterexample does not falsify either theorem, since the particular R-charge assignment restrict $W$ to particular form which violates the genericness assumption of the theorems. It is significative to find out the pattern of R-charges in such models rather than simply identify them as counterexamples with non-generic R-charges.

One feature of the R-charge assignment (2.3) is the existence of the oppositely R-charged field pair $z_{3}$ and $z_{4}$. They appear as a product $z_{3} z_{4}$ in the superpotential (2.4), although $z_{3}$ also appears linearly in another cubic term. The work of [24] explores this feature and presents a sufficient condition for counterexamples. New counterexamples are constructed using one R-charge 2 field, no R-charge 0 field, and more than one field pairs with opposite R-charges. At least one pair of oppositely R-charged fields satisfy the condition that they both appear only linearly in the superpotential, and are not involved in any quadratic term. The product of such a field pair gets a non-zero VEV, and acts effectively like an R-charge 0 field to help solving the F-term equations. It is natural to generalize the sufficient condition and the counterexample construction to the case with multiple R-charge 2 and R-charge 0 fields. Such generalization is to be done in the following section.

\section{A sufficient condition for counterexamples}

Following the convention in [11] which is also used in [2, 3], we make the following field classification in an R-symmetric Wess-Zumino model:

Definition 1 (The field classification) Under an R-symmetry, fields are classified according to their $R$-charges into the following types: 
- $X$ fields: $X_{i}$ for $i=1, \ldots, N_{X}$ with $R\left(X_{i}\right)=2$;

- $Y$ fields: $Y_{i}$ for $i=1, \ldots, N_{Y}$ with $R\left(Y_{i}\right)=0$;

- $P_{(r)}$ and $Q_{(-r)}$ fields for a value of $r: P_{(r) i}$ for $i=1, \ldots, N_{P(r)}$ and $Q_{(-r) j}$ for $j=1, \ldots, N_{Q(-r)}$ with $R\left(P_{(r) i}\right)=-R\left(Q_{(-r) j}\right)=r$, and both $P$ 's and $Q$ 's only appear linearly in the superpotential and not in any quadratic terms;

- A fields: $A_{i}$ for $i=1, \ldots, N_{A}$ with $R\left(A_{i}\right)=r_{i} \neq 2$ or 0 , and $A$ 's do not satisfy the condition for being classified as $P_{(r)}$ and $Q_{(-r)}$ fields.

For a renormalizable superpotential, both $P$ 's and $Q$ 's only appear linearly in cubic terms. Other oppositely R-charged field pairs which can not be classified as $P$ - $Q$ pairs are identified as $A$ 's. This field classification leads to the generic, R-symmetric and renormalizable superpotential

$$
\begin{aligned}
W= & W_{0}+W_{1}, \\
W_{0}= & X_{i}\left(a_{i}+b_{i j} Y_{j}+c_{i j k} Y_{j} Y_{k}+d_{(r) i j k} P_{(r) j} Q_{(-r) k}\right), \\
W_{1}= & \underbrace{\xi_{i j} X_{i}^{2} A_{j}}_{r_{j}=-2}+\underbrace{\rho_{i j k} X_{i} A_{j} A_{k}}_{r_{j}+r_{k}=0}+\underbrace{\sigma_{(r) i j k} P_{(r) i} A_{j} A_{k}}_{r_{j}+r_{k}=2-r}+\underbrace{\tau(r) i j k}_{r_{j}+r_{k}=2+r} Q_{(-r) i} A_{j} A_{k} \\
& +\underbrace{\left(\mu_{i j}+\nu_{i j k} Y_{k}\right) A_{i} A_{j}}_{r_{i}+r_{j}=2}+\underbrace{\lambda_{i j k} A_{i} A_{j} A_{k}}_{r_{i}+r_{j}+r_{k}=2} .
\end{aligned}
$$

All terms of $W_{1}$ are at least quadratic in $X$ 's and $A$ 's. This statement is also true for non-renormalizable superpotentials. So if we look for a vacuum satisfying

$$
X_{i}=A_{j}=0
$$

the first derivatives of $W_{0}$ respecting to $Y$ 's, $P$ 's, $Q$ 's and $A$ 's, as well as all first derivatives of $W_{1}$ vanish at such a vacuum. The F-term equations are then reduced to

$$
a_{i}+b_{i j} Y_{j}+c_{i j k} Y_{j} Y_{k}+d_{(r) i j k} P_{(r) j} Q_{(-r) k}=0
$$

or more generally

$$
f_{i}\left(Y_{j}, P_{(r) k} Q_{(-r) l}\right)=0
$$

which also covers the non-renormalizable case. Suppose that the total number of independent quadratic products $P_{(r) j} Q_{(-r) k}$, or the number of independent $P$ - $Q$ pairs with opposite R-charges, is $N_{P Q}$. These quadratic products can be effectively viewed as $N_{P Q}$ independent variables. So there are totally $N_{Y}+N_{P Q}$ variables to solve $N_{X}$ equations. SUSY solutions exist with $N_{X} \leq N_{Y}+N_{P Q}$ and generic parameters. But the revised theorem predicts SUSY breaking when $N_{X}>N_{Y}$. Notice also that a solution to (3.3) or (3.4) generically gives non-zero VEV's for $P$ 's and $Q$ 's, which spontaneously break the R-symmetry. Thus the generic superpotential (3.1) under the condition

$$
N_{Y}<N_{X} \leq N_{Y}+N_{P Q}
$$

gives a counterexample to both the original Nelson-Seiberg theorem and the revised one. 
The total number of $P-Q$ pairs is

$$
N_{P Q}^{\prime}=\sum_{r} N_{P(r)} N_{Q(-r)} .
$$

But the corresponding quadratic products may not be independent when there are multiple fields with degenerated R-charges. The set

$$
\bigcup_{r}\left\{P_{(r) j} Q_{(-r) k} \mid j=1 \vee k=1\right\}
$$

contains independent elements, since every quadratic product in this set except $P_{(r) 1} Q_{(-r) 1}$ has either $P_{(r) j}$ or $Q_{(-r) k}$ not appearing in other set elements. Quadratic products not in (3.7), if existing, can be expressed in terms of the elements of (3.7):

$$
P_{(r) j} Q_{(-r) k}=\frac{\left(P_{(r) j} Q_{(-r) 1}\right)\left(P_{(r) 1} Q_{(-r) k}\right)}{P_{(r) 1} Q_{(-r) 1}}, \quad j, k>1 .
$$

Thus the order of the set (3.7) gives the number of independent $P$ - $Q$ pairs

$$
N_{P Q}=\sum_{r}\left(N_{P(r)}+N_{Q(-r)}-1\right) .
$$

Notice that $N_{P Q}<N_{P Q}^{\prime}$ if there is at least one $r$ with $N_{P(r)}>1$ and $N_{Q(-r)}>1$.

In summary, we have obtained the sufficient condition:

Theorem 3 (A sufficient condition for counterexamples to the Nelson-Seiberg theorem) In a Wess-Zumino model with a generic $R$-symmetric superpotential, using the notation in Definition 1, the condition $N_{Y}<N_{X} \leq N_{Y}+N_{P Q}$ is a sufficient condition for the model to be a counterexample to both the original Nelson-Seiberg theorem and the revised one, where $N_{P Q}=\sum_{r}\left(N_{P(r)}+N_{Q(-r)}-1\right)$ is the number of independent P-Q pairs.

The total number of $P$ 's and $Q$ 's is

$$
N_{P+Q}=\sum_{r}\left(N_{P(r)}+N_{Q(-r)}\right) .
$$

The reduced F-term equations (3.3) or (3.4) can also be viewed as using $N_{Y}+N_{P+Q}$ variables to solve $N_{X}$ equations. Generically each solution has $N_{Y}+N_{P+Q}-N_{X}$ undetermined variables, or degeneracy of complex dimension $N_{Y}+N_{P+Q}-N_{X}$. Since $N_{P+Q}$ is greater than $N_{P Q}$ for models with at least one $P-Q$ pairs, SUSY solutions in counterexamples have degeneracy dimension of at least one. The VEV's of $P$ 's and $Q$ 's are generically non-zero, and the R-symmetry is spontaneously broken everywhere on the degenerated vacuum. Such a SUSY solution also makes the superpotential (3.1) to be zero, thus the bound on the superpotential is satisfied $[29,30]$. These properties of vacua from counterexample models are summarized in the following theorem:

Theorem 4 (Properties of vacua from counterexamples) A counterexample model according to Theorem 3 gives one or several SUSY vacua with $W=0$ and degeneracy of complex dimension $N_{Y}+N_{P+Q}-N_{X}$, where $N_{P+Q}=\sum_{r}\left(N_{P(r)}+N_{Q(-r)}\right)$ is the total number of $P$ 's and $Q$ 's. The R-symmetry is spontaneously broken by the non-zero VEV's of P-Q pairs everywhere on the degenerated vacua. 


\section{Examples of counterexamples}

The sufficient condition is demonstrated by the following examples of counterexample models. The simplest counterexample (2.3) and (2.4) can be rewritten using the notation in Definition 1, as the R-charge assignment

$$
(R(X), R(P), R(Q), R(A))=(2,6,-6,-2)
$$

and the superpotential

$$
W=X(a+d P Q)+\xi X^{2} A+\sigma P A^{2} .
$$

It has $N_{X}=N_{P(6)}=N_{Q(-6)}=1, N_{Y}=0$, and thus $N_{P Q}=1$. The quadratic product $P Q$, viewed effectively as an $\mathrm{R}$-charge 0 variable, solves the reduced $\mathrm{F}$-term equation $a+d P Q=0$. The SUSY solution is given as

$$
X=A=0, \quad P Q=-\frac{a}{d},
$$

and the R-symmetry is broken by the non-zero VEV's of $P$ and $Q$.

As an example of models with both $Y$ 's and $P$ - $Q$ pairs, the R-charge assignment

$$
\left(R\left(X_{1}\right), R\left(X_{2}\right), R(Y), R(P), R(Q), R(A)\right)=(2,2,0,6,-6,-2)
$$

leads to the superpotential

$$
\begin{aligned}
W= & X_{1}\left(a_{1}+b_{1} Y+c_{1} Y^{2}+d_{1} P Q\right)+X_{2}\left(a_{2}+b_{2} Y+c_{2} Y^{2}+d_{2} P Q\right) \\
& +\xi_{1} X_{1}^{2} A+\xi_{2} X_{2}^{2} A+\sigma P A^{2} .
\end{aligned}
$$

This model has $N_{X}=2, N_{Y}=N_{P(6)}=N_{Q(-6)}=1$, and thus $N_{P Q}=1$. The two independent variables $P Q$ and $Y$ solve the two reduced F-term equations. The SUSY solution is given as

$$
\begin{aligned}
X_{1} & =X_{2}=A=0, \quad Y=\frac{-b_{1} d_{2}+b_{2} d_{1} \pm \sqrt{\Delta}}{2\left(c_{1} d_{2}-c_{2} d_{1}\right)}, \\
P Q & =\frac{a_{1} c_{2}-a_{2} c_{1}}{c_{1} d_{2}-c_{2} d_{1}}+\frac{\left(b_{1} c_{2}-b_{2} c_{1}\right)\left(-b_{1} d_{2}+b_{2} d_{1} \pm \sqrt{\Delta}\right)}{2\left(c_{1} d_{2}-c_{2} d_{1}\right)^{2}}, \\
\text { where } \Delta & =\left(b_{1} d_{2}-b_{2} d_{1}\right)^{2}-4\left(a_{1} d_{2}-a_{2} d_{1}\right)\left(c_{1} d_{2}-c_{2} d_{1}\right),
\end{aligned}
$$

and the R-symmetry is broken by the non-zero VEV's of $P$ and $Q$.

As an example of models with some oppositely R-charged field pairs which can not be classified as $P-Q$ pairs, the R-charge assignment

$$
\left(R(X), R(P), R(Q), R\left(A_{1}\right), R\left(A_{2}\right)\right)=(2,4,-4,1,-1)
$$

leads to the superpotential

$$
W=X(a+d P Q)+\rho X A_{1} A_{2}+\sigma P A_{2}^{2}+\mu A_{1}^{2} .
$$


The $A_{1}-A_{2}$ pair, although with opposite R-charges, can not be classified as $P$ 's and $Q$ 's, because both $A_{1}$ and $A_{2}$ appear quadraticly in $W$, and $A_{1}$ appears in a quadratic term. This model has $N_{X}=N_{P(4)}=N_{Q(-4)}=1, N_{Y}=0$, and thus $N_{P Q}=1$. The SUSY solution is given as

$$
X=A_{1}=A_{2}=0, \quad P Q=-\frac{a}{d},
$$

and the R-symmetry is broken by the non-zero VEV's of $P$ and $Q$.

As an example of models containing multiple $P-Q$ pairs with degenerated R-charges, the R-charge assignment

$$
\left(R\left(X_{1}\right), R\left(X_{2}\right), R\left(X_{3}\right), R\left(P_{1}\right), R\left(P_{2}\right), R\left(Q_{1}\right), R\left(Q_{2}\right), R(A)\right)=(2,2,2,6,6,-6,-6,-2)
$$

leads to the superpotential

$$
\begin{aligned}
W= & X_{1}\left(a_{1}+d_{111} P_{1} Q_{1}+d_{112} P_{1} Q_{2}+d_{121} P_{2} Q_{1}+d_{122} P_{2} Q_{2}\right) \\
& +X_{2}\left(a_{2}+d_{211} P_{1} Q_{1}+d_{212} P_{1} Q_{2}+d_{221} P_{2} Q_{1}+d_{222} P_{2} Q_{2}\right) \\
& +X_{3}\left(a_{3}+d_{311} P_{1} Q_{1}+d_{312} P_{1} Q_{2}+d_{321} P_{2} Q_{1}+d_{322} P_{2} Q_{2}\right) \\
& +\xi_{1} X_{1}^{2} A+\xi_{2} X_{2}^{2} A+\xi_{3} X_{3}^{2} A+\sigma_{1} P_{1} A^{2}+\sigma_{2} P_{2} A^{2}
\end{aligned}
$$

This model has $N_{X}=3, N_{Y}=0, N_{P(6)}=N_{Q(-6)}=2$, and thus

$$
N_{P Q}=N_{P(6)}+N_{Q(-6)}-1=3 .
$$

SUSY solutions correspond to

$$
X_{1}=X_{2}=X_{3}=A=0
$$

and the solutions to

$$
\begin{aligned}
& a_{1}+d_{111} P_{1} Q_{1}+d_{112} P_{1} Q_{2}+d_{121} P_{2} Q_{1}+d_{122} P_{2} Q_{2}=0, \\
& a_{2}+d_{211} P_{1} Q_{1}+d_{212} P_{1} Q_{2}+d_{221} P_{2} Q_{1}+d_{222} P_{2} Q_{2}=0, \\
& a_{3}+d_{311} P_{1} Q_{1}+d_{312} P_{1} Q_{2}+d_{321} P_{2} Q_{1}+d_{322} P_{2} Q_{2}=0 .
\end{aligned}
$$

The three independent variables $P_{1} Q_{1}, P_{1} Q_{2}$ and $P_{2} Q_{1}$ solve the three reduced F-term equations. Then $P_{1}, P_{2}, Q_{1}$ and $Q_{2}$ can be expressed in terms of these three variables and a free parameter representing the degeneracy. The analytical solution is a radical expression of several hundred terms, which is too complicated to be presented here. Numerical solutions with some typical choices of coefficient values are listed in table 1 . These solutions, together with (4.13), give SUSY vacua with R-symmetry breaking by the non-zero VEV's of $P_{1}, P_{2}, Q_{1}$ and $Q_{2}$.

All models in this section satisfy $N_{Y}<N_{X} \leq N_{Y}+N_{P Q}$, thus are counterexamples to the Nelson-Seiberg theorem according to Theorem 3. The analytically or numerically obtained SUSY solutions have $W=0$, degeneracy of $N_{Y}+N_{P+Q}-N_{X}$, and R-symmetry breaking everywhere on the degenerated vacua. So both Theorem 3 and Theorem 4 are verified by these examples. 


\begin{tabular}{|c|c|c|}
\hline$\left(a_{1}, d_{111}, d_{112}, d_{121}, d_{122}\right)$ & $\left(a_{2}, d_{211}, d_{212}, d_{221}, d_{222}\right)$ & $\left(a_{3}, d_{311}, d_{312}, d_{321}, d_{322}\right)$ \\
\hline$(1.2,-1.3,1.8,1.3,-0.8)$ & $(-0.6,0.9,1.2,1.8,0.7)$ & $(-1.9,-1.6,-1.1,-1.2,1.1)$ \\
\hline \multicolumn{4}{|c|}{$P_{1} Q_{1}=-0.267532, P_{2}=-1.73027 P_{1}, Q_{2}=2.52332 Q_{1}$} \\
or $P_{1} Q_{1}=-0.0503819, P_{2}=7.04817 P_{1}, Q_{2}=-4.15665 Q_{1}$ \\
\hline$(-1.7,-1.1,1.5,-0.8,1.4)$ & $(-1.2,1.7,0.5,1.9,0.6)$ & $(1.7,-1.9,-1.5,-0.6,0.7)$ \\
\hline \multicolumn{4}{|c|}{$P_{1} Q_{1}=-5.96733, P_{2}=-1.04776 P_{1}, Q_{2}=-0.696789 Q_{1}$} \\
or $P_{1} Q_{1}=0.208592, P_{2}=0.345409 P_{1}, Q_{2}=4.80255 Q_{1}$ \\
\hline$(0.8,-1.6,0.7,-0.6,-1.8)$ & $(-1.6,-1.5,-0.5,-1.7,0.9)$ & $(-1.7,0.9,-0.6,1.9,-1.7)$ \\
\hline \multicolumn{4}{|c|}{$P_{1} Q_{1}=-3.50319, P_{2}=-1.01492 P_{1}, Q_{2}=0.48258 Q_{1}$} \\
or $P_{1} Q_{1}=-0.320605, P_{2}=0.106889 P_{1}, Q_{2}=8.19428 Q_{1}$ \\
\hline$(-0.6,0.8,-1.3,-1.4,-1.1)$ & $(-1.6,-1.3,-1.4,1.8,-0.7)$ & $(1.3,-0.6,-1.7,1.7,0.6)$ \\
\hline \multicolumn{4}{|c|}{$P_{1} Q_{1}=-4.32013, P_{2}=0.581242 P_{1}, Q_{2}=0.064529 Q_{1}$} \\
or $P_{1} Q_{1}=-0.14664, P_{2}=-3.28734 P_{1}, Q_{2}=-4.09916 Q_{1}$ \\
\hline
\end{tabular}

Table 1. Numerical solutions to (4.14) with typical choices of coefficient values.

\section{Outlook}

In this work, we investigate features of counterexamples to the Nelson-Seiberg theorem, and successfully proved a theorem which provides a sufficient condition for counterexamples. The scope of the theorem covers all previously found counterexamples in literature [23, 24]. The sufficient condition is expressed as the comparison between field counts of different $\mathrm{R}$ charges, and the count of independent $P-Q$ pairs has the simple expression (3.9). It is still feasible to do a fast survey of a large number of models using the field counting method, even taking into account the counterexamples in this work. Thus the pattern of field counts in counterexamples enables a refined classification of R-symmetric Wess-Zumino models.

Counterexample models built from our sufficient condition have certain properties, which may inspire applications in both phenomenology and formal studies. The R-symmetry breaking SUSY vacua are applicable to model building of tree-level R-symmetry breaking [31-34], where the R-symmetry breaking sector can be build separately from the SUSY breaking sector. The SUSY vacua with $W=0$ become Minkowski SUSY vacua in supergravity, which make up the low-energy SUSY branch of the string landscape [35-37]. The degeneracy direction of vacua can be used for cosmological model building with non-perturbative potentials introduced, and the difficulty from the de Sitter swampland conjecture may be avoided [38-41]. It is still challenging to find ultraviolet completion of these models in strongly coupled SUSY gauge theories or compactification of string theory. 


\section{Acknowledgments}

We thank Yan He and Jinmian Li for helpful discussions. This work is supported by the National Natural Science Foundation of China under grant 11305110.

Open Access. This article is distributed under the terms of the Creative Commons Attribution License (CC-BY 4.0), which permits any use, distribution and reproduction in any medium, provided the original author(s) and source are credited.

\section{References}

[1] A.E. Nelson and N. Seiberg, $R$ symmetry breaking versus supersymmetry breaking, Nucl. Phys. B 416 (1994) 46 [hep-ph/9309299] [InSPIRE].

[2] Z. Kang, T. Li and Z. Sun, The Nelson-Seiberg theorem revised, JHEP 12 (2013) 093 [arXiv: 1209.1059] [INSPIRE].

[3] Z. Li and Z. Sun, The Nelson-Seiberg theorem generalized with nonpolynomial superpotentials, Adv. High Energy Phys. 2020 (2020) 3701943 [arXiv: 2006. 00538] [INSPIRE].

[4] K.A. Intriligator and N. Seiberg, Lectures on Supersymmetry Breaking, Class. Quant. Grav. 24 (2007) S741 [hep-ph/0702069] [INSPIRE].

[5] J. Wess and B. Zumino, A Lagrangian Model Invariant Under Supergauge Transformations, Phys. Lett. B 49 (1974) 52 [INSPIRE].

[6] J. Wess and B. Zumino, Supergauge Invariant Extension of Quantum Electrodynamics, Nucl. Phys. B 78 (1974) 1 [INSPIRE].

[7] L. O'Raifeartaigh, Spontaneous Symmetry Breaking for Chiral Scalar Superfields, Nucl. Phys. B 96 (1975) 331 [INSPIRE].

[8] K.A. Intriligator, N. Seiberg and D. Shih, Dynamical SUSY breaking in meta-stable vacua, JHEP 04 (2006) 021 [hep-th/0602239] [INSPIRE].

[9] K.A. Intriligator, N. Seiberg and D. Shih, Supersymmetry breaking, R-symmetry breaking and metastable vacua, JHEP 07 (2007) 017 [hep-th/0703281] [INSPIRE].

[10] H. Abe, T. Kobayashi and Y. Omura, R-symmetry, supersymmetry breaking and metastable vacua in global and local supersymmetric theories, JHEP 11 (2007) 044 [arXiv:0708.3148] [INSPIRE].

[11] Z. Sun, Low energy supersymmetry from R-symmetries, Phys. Lett. B 712 (2012) 442 [arXiv:1109.6421] [INSPIRE].

[12] J. Brister, Z. Li and Z. Sun, Non-Abelian R-symmetries for $4 d$ N=1 supersymmetry, to appear.

[13] H.P. Nilles, Supersymmetry, Supergravity and Particle Physics, Phys. Rept. 110 (1984) 1 [INSPIRE].

[14] S.P. Martin, A Supersymmetry primer, Adv. Ser. Direct. High Energy Phys. 21 (2010) 1 [hep-ph/9709356] [INSPIRE].

[15] H. Baer and X. Tata, Weak scale supersymmetry: From superfields to scattering events, Cambridge University Press (2006) [INSPIRE]. 
[16] J. Terning, Modern supersymmetry, Oxford University Press (2006).

[17] M. Dine, Supersymmetry and String Theory, 2nd edition, Cambridge University Press (2016).

[18] M. Graña, Flux compactifications in string theory: A Comprehensive review, Phys. Rept. 423 (2006) 91 [hep-th/0509003] [INSPIRE].

[19] M.R. Douglas and S. Kachru, Flux compactification, Rev. Mod. Phys. 79 (2007) 733 [hep-th/0610102] [INSPIRE].

[20] R. Blumenhagen, B. Körs, D. Lüst and S. Stieberger, Four-dimensional String Compactifications with D-branes, Orientifolds and Fluxes, Phys. Rept. 445 (2007) 1 [hep-th/0610327] [INSPIRE].

[21] L.E. Ibanez and A.M. Uranga, String theory and particle physics: An introduction to string phenomenology, Cambridge University Press (2012).

[22] R. Blumenhagen, D. Lüst and S. Theisen, Basic concepts of string theory, Springer (2013).

[23] Z. Sun, Z. Tan and L. Yang, A counterexample to the Nelson-Seiberg theorem, JHEP 10 (2020) 072 [arXiv : 1904.09589] [InSPIRE].

[24] A. Amariti and D. Sauro, On the Nelson-Seiberg theorem: generalizations and counter-examples, arXiv:2005.02076 [INSPIRE].

[25] L. Ferretti, R-symmetry breaking, runaway directions and global symmetries in O'Raifeartaigh models, JHEP 12 (2007) 064 [arXiv:0705.1959] [INSPIRE].

[26] L. Ferretti, O'Raifeartaigh models with spontaneous R-symmetry breaking, AIP Conf. Proc. 957 (2007) 221 [arXiv:0710.2535] [INSPIRE].

[27] T. Azeyanagi, T. Kobayashi, A. Ogasahara and K. Yoshioka, Runaway, D term and R-symmetry Breaking, Phys. Rev. D 86 (2012) 095026 [arXiv: 1208.0796] [INSPIRE].

[28] Z. Sun and X. Wei, Runaway Directions in O'Raifeartaigh Models, Commun. Theor. Phys. 70 (2018) 677 [arXiv: 1806.02384] [INSPIRE].

[29] R. Kappl, H.P. Nilles, S. Ramos-Sanchez, M. Ratz, K. Schmidt-Hoberg and P.K.S. Vaudrevange, Large hierarchies from approximate R symmetries, Phys. Rev. Lett. 102 (2009) 121602 [arXiv:0812.2120] [INSPIRE].

[30] M. Dine, G. Festuccia and Z. Komargodski, A Bound on the Superpotential, JHEP 03 (2010) 011 [arXiv: 0910.2527] [INSPIRE].

[31] L.M. Carpenter, M. Dine, G. Festuccia and J.D. Mason, Implementing General Gauge Mediation, Phys. Rev. D 79 (2009) 035002 [arXiv:0805. 2944] [INSPIRE].

[32] Z. Sun, Tree level spontaneous R-symmetry breaking in O'Raifeartaigh models, JHEP 01 (2009) 002 [arXiv:0810.0477] [INSPIRE].

[33] Z. Komargodski and D. Shih, Notes on SUSY and R-Symmetry Breaking in Wess-Zumino Models, JHEP 04 (2009) 093 [arXiv: 0902.0030] [INSPIRE].

[34] F. Liu, M. Liu and Z. Sun, No-go for tree-level R-symmetry breaking, Eur. Phys. J. C 77 (2017) 745 [arXiv: 1412.0183] [INSPIRE].

[35] M. Dine, E. Gorbatov and S.D. Thomas, Low energy supersymmetry from the landscape, JHEP 08 (2008) 098 [hep-th/0407043] [INSPIRE].

[36] M. Dine, D. O'Neil and Z. Sun, Branches of the landscape, JHEP 07 (2005) 014 [hep-th/0501214] [INSPIRE]. 
[37] M. Dine and Z. Sun, R symmetries in the landscape, JHEP 01 (2006) 129 [hep-th/0506246] [INSPIRE].

[38] G. Obied, H. Ooguri, L. Spodyneiko and C. Vafa, De Sitter Space and the Swampland, arXiv: 1806.08362 [INSPIRE].

[39] S.K. Garg and C. Krishnan, Bounds on Slow Roll and the de Sitter Swampland, JHEP 11 (2019) 075 [arXiv : 1807.05193] [inSPIRE].

[40] H. Ooguri, E. Palti, G. Shiu and C. Vafa, Distance and de Sitter Conjectures on the Swampland, Phys. Lett. B $\mathbf{7 8 8}$ (2019) 180 [arXiv:1810.05506] [INSPIRE].

[41] E. Palti, The Swampland: Introduction and Review, Fortsch. Phys. 67 (2019) 1900037 [arXiv: 1903.06239] [INSPIRE]. 